\title{
AN EXTENSION OF A COVARIANT DIFFERENTIATION PROCESS ${ }^{1}$
}

\author{
MARIE M. JOHNSON
}

Craig $^{2}$ has considered tensors $T_{\beta}^{\alpha . . .}$ whose components are functions of $n$ variables represented by $x$ and their $m$ derivatives $x^{\prime}, x^{\prime \prime}, \cdots, x^{(m)}$. He obtained the covariant derivative

$$
\begin{aligned}
& T_{\beta}^{\alpha \cdots x^{(m-1) \gamma}}-m T_{\beta \cdots x^{(m) \lambda}}^{\alpha \cdots}\left\{\begin{array}{l}
\lambda \\
\gamma
\end{array}\right\}, \\
& m \geqq 2 \text {, }
\end{aligned}
$$

where

$$
\left\{\begin{array}{l}
\lambda \\
\gamma
\end{array}\right\} \equiv x^{\prime \alpha} \Gamma_{\gamma \alpha}^{\lambda}+(1 / 2) x^{\prime \prime \beta} f_{\gamma \delta \beta} f^{\delta \lambda},
$$

and partial differentiation in (1) is denoted by the added subscript. Throughout, a repeated letter in one term indicates a sum of $n$ terms. The purpose of this note is to derive another tensor from $T_{\beta}^{\alpha \ldots} \ldots$ whose covariant rank is one larger. The general process will be shown clearly by using $T^{\alpha}\left(x, x^{\prime}, x^{\prime \prime}, x^{\prime \prime \prime}\right)$.

The extended point transformation

$$
\begin{aligned}
x^{\alpha} & =x^{\alpha}(y), \quad x^{\prime \alpha}=\frac{\partial x^{\alpha}}{\partial y^{i}} y^{\prime i}, \\
x^{\prime \prime \alpha} & =\frac{\partial x^{\alpha}}{\partial y^{i}} y^{\prime \prime i}+\frac{\partial^{2} x^{\alpha}}{\partial y^{i} \partial y^{j}} y^{\prime i} y^{\prime j}, \ldots, \quad \alpha=1, \cdots, n,
\end{aligned}
$$

gives the tensor equations of transformation of the tensor $T^{\alpha}$ as

$$
\bar{T}^{i}\left(y, y^{\prime}, y^{\prime \prime}, y^{\prime \prime \prime}\right)=T^{\alpha}\left(x, x^{\prime}, x^{\prime \prime}, x^{\prime \prime \prime}\right) \partial y^{i} / \partial x^{\alpha},
$$

where $y$ stands for the $n$ variables $y^{1}, y^{2}, \cdots, y^{n}$ and a similar notation is used for the derivatives $y^{\prime}, y^{\prime \prime}$, and $y^{\prime \prime \prime}$. On differentiating equations (3) with respect to $y^{\prime k}$ it is found that

$$
\bar{T}_{y^{\prime} k}^{i}=\left(T_{x^{\prime} \beta}^{\alpha} \frac{\partial x^{\beta}}{\partial y^{k}}+T_{x^{\prime \prime} \beta}^{\alpha} \frac{\partial x^{\prime \prime \beta}}{\partial y^{\prime k}}+T_{x^{\prime \prime \prime}}^{\alpha} \frac{\partial x^{\prime \prime \beta}}{\partial y^{\prime k}}\right) \partial y^{i} / \partial x^{\alpha} .
$$

The derivatives can be expressed by using the following general formulas:

\footnotetext{
1 Presented to the Society, April 15, 1939.

${ }^{2} \mathrm{H}$. V. Craig, On a covariant differentiation process, this Bulletin, vol. 37 (1931), pp. 731-734.
} 


$$
\frac{\partial x^{(m-1) \beta}}{\partial y^{(m-2) k}}=(m-1) \frac{\partial x^{\prime \beta}}{\partial y^{k}}, \quad \frac{\partial x^{(m) \beta}}{\partial y^{(m-2) k}}=\frac{m(m-1)}{2} \frac{\partial x^{\prime \prime \beta}}{\partial y^{k}},
$$

in which $\partial x^{\prime \beta} / \partial y^{k}$ are eliminated $b y^{3}$

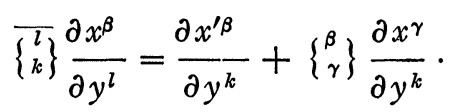

The derivatives $\partial x^{\prime \prime \beta} / \partial y^{k}$ are simplified by first writing

$$
x^{\prime \prime \beta}=\frac{\partial x^{\beta}}{\partial y^{j}} y^{\prime \prime j}+\bar{\Gamma}_{j k}^{r} y^{\prime j} y^{\prime k} \frac{\partial x^{\beta}}{\partial y^{r}}-\Gamma_{\alpha \delta}^{\beta} x^{\prime \alpha} x^{\prime \delta},
$$

with the help of (2), (6) and $f_{\alpha \beta \gamma} x^{\prime \beta}=0$. It is necessary also to have

$$
\frac{\partial^{2} x^{\beta}}{\partial y^{j} \partial y^{k}}=\bar{\Lambda}_{j k}^{t} \frac{\partial x^{\beta}}{\partial y^{t}}-\Lambda_{\alpha \delta}^{\beta} \frac{\partial x^{\alpha}}{\partial y^{j}} \frac{\partial x^{\delta}}{\partial y^{k}},
$$

where

$$
\Lambda_{\alpha \delta}^{\beta}=\Gamma_{\alpha \delta}^{\beta}-(1 / 2) f^{\beta \gamma}\left(f_{\delta \gamma \tau}\left\{\begin{array}{c}
\tau \\
\alpha
\end{array}\right\}+f_{\gamma \alpha \tau}\left\{\begin{array}{l}
\tau \\
\delta
\end{array}\right\}-f_{\alpha \delta \tau}\left\{\begin{array}{c}
\tau \\
\gamma
\end{array}\right\}\right) .
$$

This is obtained from Taylor' $\mathrm{s}^{5}$ formula (19) in the following way. Multiply this formula by $\left(\partial y^{k} / \partial x^{\epsilon}\right) f^{\beta \epsilon}=\left(\partial x^{\beta} / \partial y^{l}\right) \bar{f}^{k l}$, and sum for $k$. Use the tensor equations for $f_{\alpha \beta \gamma}$ and substitute from (6) for $\partial x^{\prime \gamma} / \partial y^{j}$.

By means of formulas (6) and (8) and the tensor $Q^{\beta}\left(x, x^{\prime}, x^{\prime \prime}\right)$ $\equiv x^{\prime \prime \beta}+\Gamma_{\alpha \delta}^{\beta} x^{\prime \alpha} x^{\prime \delta}$ the partial derivatives of (7) have the form

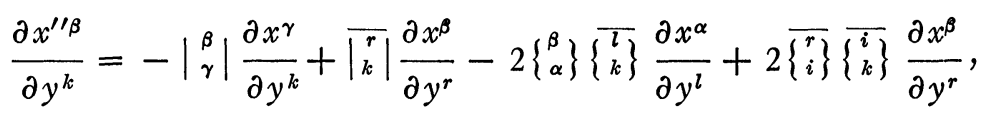

in which we have the nontensor form

$$
\left|\begin{array}{l}
\beta \\
\gamma
\end{array}\right|=Q_{x^{\gamma}}^{\beta}-Q_{x^{\prime}}^{\beta}\left\{\begin{array}{l}
\alpha \\
\gamma
\end{array}\right\}+Q^{\alpha} \Lambda_{\alpha \gamma}^{\beta} .
$$

If formulas (6) and (9) are substituted in equations (5) and the results used in (4), we find

$$
\begin{aligned}
\bar{T}_{y^{\prime, k}}^{i}= & \left(T_{x^{\prime} \beta}^{\alpha}-2 T_{x^{\prime}, \delta}^{\alpha}\left\{\begin{array}{l}
\delta \\
\beta
\end{array}\right\}-\left.3 T_{x^{\prime \prime}, \delta}^{\alpha}\right|_{\beta} ^{\delta} \mid\right) \frac{\partial x^{\beta}}{\partial y^{k}} \frac{\partial y^{i}}{\partial x^{\alpha}} \\
& -\left(-2 \bar{T}_{y^{\prime \prime l}}^{i} \overline{\left\{\begin{array}{l}
l \\
k
\end{array}\right\}}-3 \bar{T}_{y^{\prime \prime \prime l}}^{i} \overline{\mid \begin{array}{l}
l \\
k
\end{array}}\right) .
\end{aligned}
$$

${ }^{3} \mathrm{~J} . \mathrm{H}$. Taylor, A generalization of Levi-Civita's parallelism and the Frenet formulas' Transactions of this Society, vol. 27 (1925), p. 255.

${ }^{4} \mathrm{~J}$. H. Taylor, loc. cit., p. 248.

5 J. H. Taylor, loc. cit., p. 254. 
Hence the new tensor whose covariant rank has been increased by one is

$$
T_{x^{\prime \beta}}^{\alpha}-2 T_{x^{\prime \prime} \delta}^{\alpha}\left\{\begin{array}{l}
\delta \\
\beta
\end{array}\right\}-\left.3 T_{x^{\prime \prime, \delta}, \delta}^{\alpha}\right|_{\beta} ^{\delta} \mid,
$$

where $\left\{\begin{array}{l}\delta \\ \beta\end{array}\right\}$ and $\left|\begin{array}{l}\delta \\ \beta\end{array}\right|$ are defined in (2) and (10).

Because of the general relations in (5) it is easy to verify that the tensor

$$
\begin{array}{r}
T_{\gamma}^{\alpha \cdots x^{(m-2) \beta}}-(m-1) T_{\gamma}^{\alpha \cdots x^{(m-1) \delta}}\left\{\begin{array}{l}
\delta \\
\beta
\end{array}\right\}-\left.\frac{m(m-1)}{2} T_{\gamma}^{\alpha \cdots x^{(m) \delta}}\right|_{\beta} ^{\delta} \mid, \\
m \geqq 3,
\end{array}
$$

has a covariant rank which is one larger than that of $T_{\gamma}^{\alpha \ldots *}$ whose components are functions of $\left(x, x^{\prime}, \cdots, x^{(m)}\right)$.

If the components of the tensor $T^{\alpha}\left(x, x^{\prime}, x^{\prime \prime}, x^{\prime \prime \prime}\right)$ do not contain the derivatives $x^{\prime \prime \prime}$, then (11) reduces to Craig's covariant derivative (1), and if there are no $x^{\prime \prime}$ or $x^{\prime \prime \prime}$ derivatives, then the result is a partial differentiation with respect to $x^{\prime}$.

The usual rules for the derivative of a sum of tensors of the same type and rank and for the product of any tensors are preserved by this provess.

If $m=2$, a scalar $T\left(x, x^{\prime}, x^{\prime \prime}\right)$ will give a covariant tensor which is similar to that in (11) when the tensor equations for $\bar{T}\left(y, y^{\prime}, y^{\prime \prime}\right)$ are differentiated with respect to $y$ instead of $y^{\prime}$. The tensor is

$$
T_{x \beta}-T_{x^{\prime} \delta}\left\{\begin{array}{l}
\delta \\
\beta
\end{array}\right\}-\left.T_{x^{\prime}, \delta}\right|_{\beta} ^{\delta} \mid \text {. }
$$

However, if $m=2$ and a tensor $T^{\alpha}\left(x, x^{\prime}, x^{\prime \prime}\right)$ is used, an extra term $T^{\delta} \Lambda_{\delta \beta}^{\alpha}$ has to be added to three terms similar to those in (13). If this process is performed on the tensor $Q^{\alpha}\left(x, x^{\prime}, x^{\prime \prime}\right)$, the result is the zero tensor.

Oberlin College 